\title{
Possibility theory and possibilistic logic: Tools for reasoning under and about incomplete information
}

\author{
Didier Dubois and Henri Prade \\ IRIT - CNRS, 118, route de Narbonne, 31062 Toulouse Cedex 09, France \\ \{dubois, prade\}eirit.fr
}

\begin{abstract}
This brief overview provides a quick survey of qualitative possibility theory and possibilistic logic along with their applications to various forms of epistemic reasoning under and about incomplete information. It is highlighted that this formalism has the potential of relating various independently introduced logics for epistemic reasoning.
\end{abstract}

\section{Introduction}

Possibility theory is a formal setting for uncertainty management that has been independently proposed by different authors since the late 1940 's (e.g. $[63,54,27]$ ). It has been later on fully developed in the last 40 years $[66,34,40]$. It offers a framework dedicated to the representation of incomplete information, that can be naturally extended to graded beliefs, and the ensuing forms of uncertain reasoning. Because of its simplicity and natural appeal, some basic parts of this setting are implicit in many theories of epistemic reasoning, so that possibility theory has a significant unifying power for these alternative formalisms.

This extended abstract presents a short survey of possibility theory, of possibilistic logic that is based on it, and of their applications (providing detailed references in each case).

\section{Possibility theory}

Possibility theory has a remarkable situation among the settings devoted to the representation of imprecise and uncertain information.

First, it may be numerical or qualitative [36]. In the first case, possibility measures and the dual necessity measures can be regarded respectively as upper bounds and lower bounds of ill-known probabilities; they are also particular cases of Shafer [64] plausibility and belief functions respectively. In fact, possibility measures and necessity measures constitute the simplest, non trivial, imprecise probability system [65]. Second, when qualitative, possibility theory provides a natural approach to the grading of possibility and necessity modalities on finite scales $\mathcal{S}=\left\{1=\lambda_{1}>\ldots \lambda_{n}>\lambda_{n+1}=0\right\}$ where grades have an ordinal flavor. In the following, we focus on qualitative possibility theory.

In possibility theory, pieces of information, viewed as epistemic states, are represented in terms of sets or fuzzy sets viewed as possibility distributions. A possibility 
distribution $\pi$ is a mapping from a set of states, or universe of discourse, denoted by $U$, to a totally ordered scale $\mathcal{S}(\pi(u)=0$ means that state $u$ is rejected as impossible; $\pi(u)=1$ means that state $u$ is totally possible, i.e., plausible). When $\mathcal{S}=\{0,1\}, \pi$ is the characteristic function of a set $E$ of possible mutually exclusive values or states of affairs, obtained by ruling out the impossible. Such a set represents the epistemic state of an agent and is called an epistemic set. In the general, graded, case, $E$ is a fuzzy set.

Two increasing set functions, similar to probability functions, are induced from a possibility distribution, namely a possibility measure $\Pi\left(\Pi(A)=\sup _{u \in A} \pi(u)\right)$ and a dual necessity measure $N\left(N(A)=1-\Pi\left(A^{c}\right)\right.$, where $A^{c}=U \backslash A$ is the complement of $A) . \Pi(A)$ evaluates to what extent $A$ is consistent with the epistemic state $E$, and $N(A)$ to what extent $A$ is certainly implied by $E$. Note that $N(A)>0$ implies $\Pi(A)=1$.

In the Boolean case, when the epistemic state $E$ is a crisp subset of $U$, possibility and necessity functions are such that:

- $\Pi(A)=1$ if $A \cap E \neq \emptyset$, and 0 otherwise; so $\Pi(A)=0$ when proposition $A$ is incompatible with the epistemic state.

- $N(A)=1$ if $E \subseteq A$, and 0 otherwise; so $N(A)=1$ when proposition $A$ is true in all states of the world compatible with the epistemic state.

- When $\Pi(A)=1$ and $N(A)=0$ corresponds to the case when the epistemic state $E$ does not allow for deciding whether $A$ is true or false (ignorance).

Possibility measures satisfy a characteristic "maxitivity" property in the form of the identity $\Pi(A \cup B)=\max (\Pi(A), \Pi(B))$, and necessity measures a "minitivity" property $N(A \cap B)=\min (N(A), N(B))$.

In contrast with the minitivity of necessity functions and the maxitivity of possibility functions, it is generally not true that $\Pi(A \cap B)=\min (\Pi(A), \Pi(B))$, nor that $N(A \cup B)=\max (N(A), N(B))$; for instance let $B=A^{c}$, and $\Pi(A)=\Pi\left(A^{c}\right)=1$ (ignorance case), then $\Pi(A \cap B)=0 \neq \min (\Pi(A), \Pi(B))=1$. The property $\Pi(A \cap B)=\min (\Pi(A), \Pi(B))$ may hold for some special pair of events for instance when the possibility distribution is multidimensional, i.e., $U=U_{1} \times \cdots \times U_{k}$ and $\pi=\min _{i=1}^{k} \pi_{i}$, where $\pi_{i}$ is a marginal possibility distribution on $U_{i}$. $\pi$ is said to be decomposable and the variables are said to be non-interactive. Non-interactivity is a graded generalisation of logical independence.

Two other decreasing set functions can be associated with $\pi$ :

i) a measure of guaranteed possibility or strong possibility [36]: $\Delta(A)=\inf _{u \in A} \pi(u)$ which estimates to what extent all states in $A$ are possible according to evidence. $\Delta(A)$ can be used as a degree of evidential support for $A$;

ii) its dual conjugate $\nabla(A)=1-\Delta\left(A^{c}\right)$ : it evaluates a degree of potential or weak necessity of $A$, as it is 1 only if some state $u$ out of $A$ is impossible.

In the Boolean case, it reduces to $\Delta(A)=1$ if $A \subseteq E$ and 0 otherwise, while $\nabla(A)=1$ if $A \cup E \neq U$. Interestingly enough, the four set function are necessary for describing the relative positions of two subsets such as $A$ and $E$, see, e.g., [38]. The four set functions are weakly related by the constraint for all $A, \max (N(A), \Delta(A)) \leq$ $\min (\Pi(A), \nabla(A))$ (provided that $\pi$ and $1-\pi$ are both normalized, i.e. they reach 1 for some $u \in U)$. 


\section{Connections between possibility theory and other representations of incomplete knowledge}

Necessity and possibility functions reminds of modal logics, where these modalities, respectively denoted by $\square$ and $\diamond$, are used to prefix logical sentences. In KD modal logics, which are also known as epistemic logics [49], asserting $\square \phi$ stands for declaring that an agent believes or knows that proposition $\phi$ is true. If $\phi$ is a propositional formula that is true for those and only those states of affairs in the set $A$, asserting $\square \phi$ is faithfully encoded by the identity $N(A)=1$. Counterparts of properties of possibility functions are valid in KD modal logics, in particular minitivity of necessity functions $(\square(\phi \wedge \psi) \equiv \square \phi \wedge \square \psi)$, duality ( $\diamond \phi$ stands for $\neg \square \neg \phi$ and encodes the identity $\Pi(A)=1)$, and maxitivity of possibility functions $(\diamond(\phi \wedge \psi) \equiv \diamond \phi \wedge \diamond \psi)$. These possibilistic semantics are the basis of a very simple modal logic (MEL [3]). However, the language of modal logics is more complex than the one of possibility theory because propositions prefixed by modalities may contain modalities. The semantics is usually in terms of relations rather than epistemic states [48].

A semantics of Kleene three-valued logic can be devised in terms of multidimensional possibility distributions over a universe that is a Cartesian product $U=U_{1} \times$ $\cdots \times U_{k}$, where each subspace $U_{i}=\left\{a_{i}, \neg a_{i}\right\}$. Kleene logic uses 3 truth-values $\{T>I>F\}$. Assigning $T$ (resp. $F$ ) to an atom $a_{i}$, i.e., $\left.t\left(a_{i}\right)=T\right)\left(\right.$ resp. $t\left(a_{i}\right)=F$ ) expresses that $a_{i}$ is surely true, i.e., $N^{T}\left(a_{i}\right)=1$ (resp. surely false : $N^{F}\left(\neg a_{i}\right)=1$ ) which corresponds to Boolean possibility distributions $\pi_{i}^{T}\left(a_{i}\right)=1, \pi_{i}^{T}\left(\neg a_{i}\right)=0$ (resp. $\pi_{i}^{F}\left(a_{i}\right)=0, \pi_{i}^{F}\left(\neg a_{i}\right)=1$ ); finally assigning $I$ to $a_{i}$ expresses a lack of knowledge about whether $a_{i}$ is true or false $\left(\pi_{i}^{I}\left(a_{i}\right)=1, \pi_{i}^{I}\left(\neg a_{i}\right)=1\right)$. In Kleene logic, knowledge is thus expressed on atoms only, and it handles epistemic states in the form of possibility distributions $\min _{i=1}^{k} \pi_{i}$ where $\pi_{i} \in\left\{\pi_{i}^{T}, \pi_{i}^{F}, \pi_{i}^{I}\right\}$. A truth assignment $t$ is a partial model of the form $\left(\wedge_{i: t\left(a_{i}\right)=T} a_{i}\right) \wedge\left(\wedge_{j: t\left(a_{j}\right)=F} \neg a_{j}\right)$ encoded by the possibility distribution $\pi_{t}=\min \left(\min _{i: t\left(a_{i}\right)=T} \pi_{i}^{T}, \min _{i: t\left(a_{i}\right)=F} \pi_{i}^{F}\right)\left(\pi_{i}^{I}\right.$ disappears, being equal to 1 everywhere). The truth-functionality of Kleene logic can be justified in terms of the non-interactivity of the Boolean variables. The possibilistic framework highlights the limited expressiveness of Kleene 3-valued logic, which cannot account for epistemic states modelled by subsets of $U$ that are not partial models [26].

\section{Possibilistic logic and its applications}

Possibilistic logic (PL) [31, 39, 41, 42] amounts to a classical logic handling of certaintyqualified statements. Certainty is estimated in the setting of possibility theory as a lower bound of a necessity set-function. An elementary possibilistic formula $(a, \alpha)$ is made of a classical logic formula $a$ associated with a certainty level $\alpha \in \mathcal{S} \backslash\{0\}$. Basic PL handles only conjunctions of such formulas, and PL bases can be viewed as classical logic bases layered in terms of certainty. Semantics is in terms of epistemic states represented by fuzzy sets of interpretations. A PL base $\Gamma$ is associated with an inconsistency level above which formulas are safe from inconsistency (this level is defined by $\operatorname{inc}(\Gamma)=\max \{\alpha \mid \Gamma \vdash(\perp, \alpha)\}$, which semantically corresponds to the lack of normalization of the possibility distribution associated with $\Gamma$ ). 
Applications of possibilistic logic (and possibility theory) include

- Bayesian-like possibilistic networks [15, 12], where possibilistic conditioning is defined using a Bayesian-like equation of the form $\Pi(B \cap A)=\Pi(B \mid A) \star \Pi(A)$ where $\Pi(A)>0$ and $\star$ is the minimum in the qualitative case or the product in the quantitative case; moreover $N(B \mid A)=1-\Pi\left(B^{c} \mid A\right)$. Several notions of independence make sense in the possibilistic setting [29, 11]. Like joint probability distributions, joint possibility distributions can be decomposed into a conjunction of conditional possibility distributions (using $\star=$ minimum, or product), once an ordering of the variables is chosen, in a way similar to Bayes nets. Moreover, possibilistic nets can be directly translated into PL bases and vice-versa.

- Reasoning with default rules $[17,18]$ : Possibility theory can be used for describing the normal course of things. A default rule "if $a$ then generally $b$ " is understood formally as the constraint $\Pi(a \wedge b)>\Pi(a \wedge \neg b)$ on a possibility measure $\Pi$ describing the semantics of the available knowledge. It expresses that in the context where $a$ is true it is more possible that $b$ is true than the opposite. A default rule "if $a_{i}$ then generally $b_{i}$ " in a conditional knowledge base can be turned into a PL clause $\left(\neg a_{i} \vee b_{i}, N\left(\neg a_{i} \vee b_{i}\right)\right)$, where the necessity $N$ is computed from the set of constraints corresponding to the default rules in the base. We thus obtain a PL base encoding this set of rules. Then using PL inference on the PL base, augmented with propositional formulas describing a factual situation, enables us to perform non monotonic reasoning in agreement with a postulate-based approach (namely, rational closure in the sense of Lehmann and Magidor [53]).

- Belief revision. Since non monotonic reasoning and belief revision can be closely related, PL finds application also in belief revision. In fact, comparative necessity relations (a relational counterpart of qualitative necessity measures) [28] are nothing but the epistemic entrenchment relations [35] that underly well-behaved belief revision processes [47]. This enables the PL setting to provide syntactic revision operators that apply to possibilistic knowledge bases, including the case of uncertain inputs $[21,59]$.

- Information fusion. The combination of possibility distributions, by means of fuzzy set connectives such as min, can be equivalently performed syntactically in terms of PL bases [20]. Besides, this approach can be also applied to the syntactic encoding of the merging of classical logic bases based on Hamming distance (where distances are computed between each interpretation and the different classical logic bases, thus giving birth to counterparts of possibility distributions) [16].

- Preference modeling. In this case, certainty is turned into priority: Each PL formula $(a, \alpha)$ represents a goal $a$ to be reached with some priority level $\alpha$. Beyond PL, interpretations (corresponding to different alternatives) can be compared in terms of vectors acknowledging the satisfaction or the violation of the formulas associated with the different goals, using suitable order relations. Thus, partial orderings of interpretations can be obtained [13].

- Modeling desires: In contrast with static beliefs, (positive) desires are such that endorsing $a \vee b$ as a desire means to desire $a$ and to desire $b$. However, desiring both $a$ and $\neg a$ does not sound rational. The modeling of desires can be achieved using a "desirability" distribution $\delta: U \rightarrow[0,1]$ such that $\delta(u)=0$ for some 
$u \in U$. The logic of desires is thus a inversed mirror image of classical logic. Just as belief revision relies on an epistemic entrenchment relation (and thus on a necessity measure), well-behaved desire revision relies on a guaranteed possibility function $\Delta[33]$.

- Qualitative decision. Possibility theory provides a valuable setting for qualitative decision under uncertainty where pessimistic and optimistic decision criteria have been axiomatized [44] and cast in possibilistic logic by means of two bases, one for expressing knowledge, the other for expressing goals [32].

Let us also briefly mention different extensions of basic PL, where

- Lattice-valued possibilistic logic. Examples are i) a timed PL with logical formulas associated with fuzzy sets of time instants where the formula is known to be certain to some extent; ii) a logic of supporters [52], where formulas $a$ are associated with sets of arguments in their favor. Closely related to this latter logic is the idea of associating each formula with a set of distinct explicit sources that support its truth more or less strongly. This has led to the proposal of a "social" logic where formulas are of the form $(a, A)$, where $A$ denotes a subset of agents and the formula means that at least all the agents in $A$ believe that $a$ is true [6]. It can be extended to pieces of information of the form "at least all agents in $A$ believe $a$ at least at level $\alpha$ ".

- Symbolic PL. Instead of using weights from a totally ordered scale, one may use pairs ( $\mathrm{p}, \mathrm{x})$ where $\mathrm{x}$ is a symbolic entity that stands for an unknown weight. Then we can model the situation where only a partial ordering between ill-known weights is specified by means of inequality constraints [9,22].

- An extension of possibilistic inference has been proposed for handling paraconsistent (conflicting) information [19].

In a computational perspective, possibilistic logic has also impacted logic programming $[1,56,57,5]$. Besides, the possibilistic handling of uncertainty in description logic has been suggested [60,67]. Computational advantages of description logic can then be preserved for its PL extensions, in particular in the case of the possibilistic DL-Lite family [7, 8]. Another application is the encoding of control access policies [14].

\section{Generalized possibilistic logic}

In the so-called generalized possibilistic logic (GPL) [46], negation and disjunction can be used to combine possibilistic formulas, on top of conjunction as in PL. GPL use graded necessity and possibility modalities, i.e., a PL formula $(a, \alpha)$, is encoded as $\square_{\alpha} a$ in GPL.

GPL can be viewed as both a generalization of PL and a generalization of MetaEpistemic Logic (MEL) [3], the simplest logic of belief and partial ignorance - a fragment of modal logic KD where all formulas are modal, and modalities cannot be nested. See Figure 1 [41], which points out how GPL extends propositional logic through MEL and PL. GPL is in fact just a two-tiered standard propositional logic, in which propositional formulas are encapsulated by weighted modal operators, forming higher order propositional formulas, interpreted in terms of uncertainty measures from possibility 


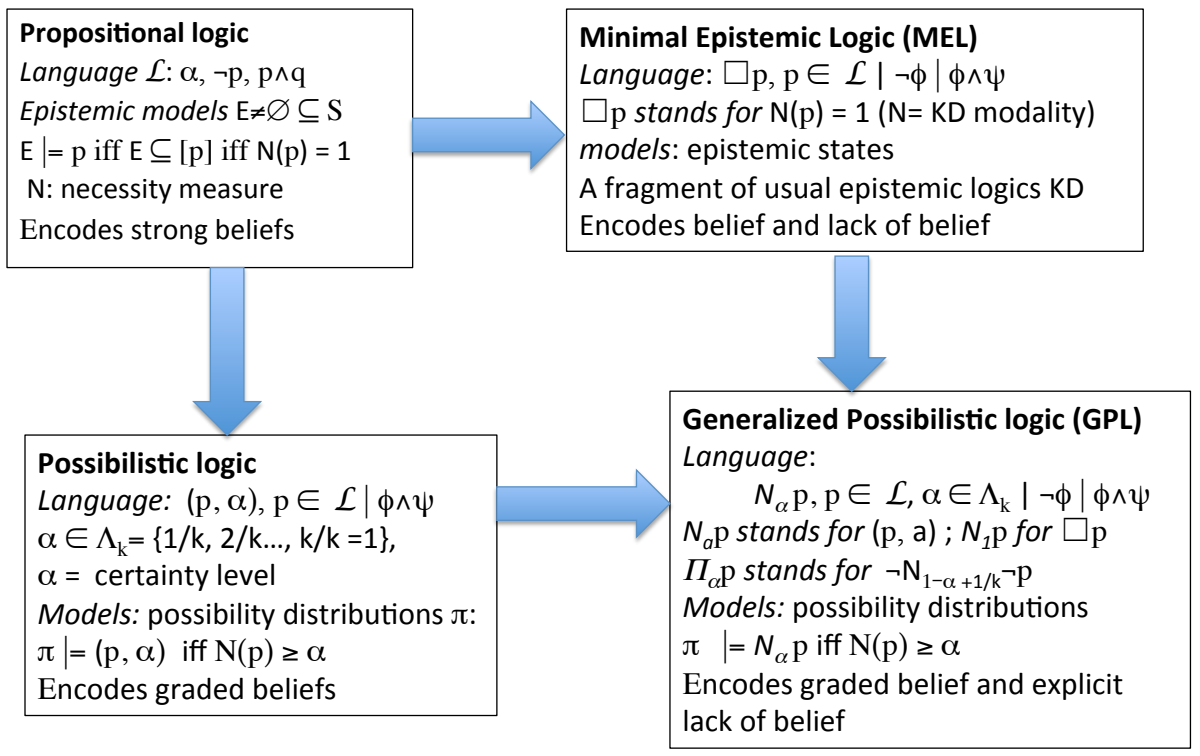

Fig. 1: From Propositional Logic to Generalized Possibilistic Logic

theory. GPL can be still extended to a logic involving both objective and non-nested multimodal formulas [4].

GPL has applications such as:

- Reasoning about ignorance [46]: Some GPL formulas encode statements such as "All that is known is $a$ ". It means that $a$ is known but nothing more (like, e.g., some $b$ entailing $a$ ). It semantically corresponds to the use of the guaranteed possibility set function.

- Representing answer set programs [45, 46]: GPL can encode answer set programs, adopting a three-valued scale $\mathcal{S}=\{0,1 / 2,1\}$. In this case, we can discriminate between propositions we are fully certain of and propositions we consider only more plausible than not. This is sufficient to encode non-monotonic ASP rules (with negation as failure) within GPL, which lays bare their epistemic semantics.

- Handling comparative certainty logic [23]. Rather than using weighted formulas, one might wish to represent at a syntactic level statements of the form " $a$ is more certain than $b$ ". This kind of statements can be to some extent captured by GPL [46], which is thus more expressive than PL with symbolic weights.

- Encoding many-valued logics of uncertainty, such as Kleene and Łukasiewicz 3valued logics [24], as well as 3-valued paraconsistent logics such as Priest logic 
of paradox [25]. MEL is enough for this task. GPL with 2 necessity modalities (one weak and one strong) is needed to encode the 5-valued equilibrium logic [45]. Many-valued logics with more truth-values could be encoded in GPL with a suitable number of certainty levels.

As a perspective, a logic of arguments, similar to GPL, has been outlined in [2]. The basic formulas are pairs $(x, y)$ and stand for " $y$ is a reason for believing $x$ ". Another perspective is reasoning about other agents' beliefs as in the muddy children problem [42].

\section{Concluding remarks}

Generally speaking, the interest and the strength of PL and GPL relies on a sound alliance between classical logic and possibility theory which offers a rich representation setting allowing an accurate modeling of partial ignorance.

An interesting feature of possibility theory (involving the four set functions) lies in its capability of providing a bipolar representation of positive and negative information using pairs of distributions $(\delta, \pi)$. This view applies both to knowledge and preferences. Then, the distribution $\pi$ describes the complement of the fuzzy set of values that are ruled out (being impossible or rejected), while the distribution $\delta$ describes a fuzzy subset of values that are actually possible to some extent or desired (positive information) [37].

Counterparts of the four possibility theory set functions also make sense in formal concept analysis, where formal concepts in the usual sense are defined from the counterpart of the $\Delta$ function and the scale $\mathcal{S}$ is replaced by a power set [38].

Possibility theory still has several other noticeable applications such as the design and the handling of uncertain databases $[58,55]$, or the numerical computation with fuzzy intervals [30].

Lastly, possibility theory may have some potential in machine learning both on the quantitative side due to its link with imprecise probabilities and the existence of probability / possibility transformations, and on the qualitative side for its relation to logic and its use in the modeling of different types of if-then rules, which may be also of interest for explanation purposes; see [43] for a discussion and references. This is still to be further explored. Let us more particularly mention some works on the qualitative side in relation with possibilistic logic. PL can be applied to inductive logic programming (ILP). Indeed having a stratified set of first-order logic rules as an hypothesis in ILP is of interest for learning both rules covering normal cases and more specific rules for exceptional cases [62]. A different approach to the induction of possibilistic logic theories is proposed in [51]. It relies on the fact that any set of formulas in Markov logic [61] can be exactly translated into possibilistic logic formulas [50,46].

\section{References}

1. T. Alsinet, L. Godo, S. Sandri. Two formalisms of extended possibilistic logic programming with context-dependent fuzzy unification: a comparative description. Elec. Notes in Theor. Computer Sci. 66 (5), 2002. 
2. L. Amgoud, H. Prade. Towards a logic of argumentation. Proc. 6th Int. Conf. on Scalable Uncertainty Management (SUM'12), Marburg, Springer LNCS, 7520, 558-565, 2012.

3. M. Banerjee, D. Dubois. A simple logic for reasoning about incomplete knowledge. Int. J. Approx. Reasoning, 55, 639-653, 2014.

4. M. Banerjee, D. Dubois, L. Godo, H. Prade. On the relation between possibilistic logic and modal logics of belief and knowledge. J. Appl. Non-Class. Log. 27(3-4), 206-224, 2017.

5. K. Bauters, S. Schockaert,M. DeCock, D. Vermeir. Possible and necessary answer sets of possibilistic answer set programs. Proc.24th IEEE Int. Conf. ICTAI, Athens, 836-843, 2012.

6. A. Belhadi, D. Dubois, F. Khellaf-Haned, H. Prade. Multiple agent possibilistic logic. J. of Applied Non-Classical Logics, 23, 299-320, 2013.

7. S. Benferhat, Z. Bouraoui. Possibilistic DL-Lite. Proc. 7th Int. Conf. SUM'13, Wash- ington, Springer LNCS 8078, 346-359, 2013.

8. S. Benferhat, Z. Bouraoui, Z. Loukil. Min-based fusion of possibilistic DL-Lite knowledge bases. Proc. IEEE/WIC/ACM Int. Conf. on Web Intelligence (WI'13), Atlanta, 23-28, 2013.

9. S. Benferhat, H. Prade. Encoding formulas with partially constrained weights in a possibilistic-like many-sorted propositional logic. Proc. 9th IJCAI, Edinburgh, 1281-1286, 2005.

10. L. Boldrin, C. Sossai. Local possibilistic logic. J. Appl. Non-Class. Log.7, 309-333, 1997.

11. N. Ben Amor, S. Benferhat, D. Dubois, K. Mellouli, H. Prade. A theoretical framework for possibilistic independence in a weakly ordered setting. Int. J. Uncertainty, Fuzziness \& Knowledge-based Syst. 10, 117-155, 2002.

12. N. Ben Amor, S. Benferhat, K. Mellouli. Anytime propagation algorithm for min-based possibilistic graphs. Soft Comput. 8(2), 150-161, 2003.

13. N. Ben Amor, D. Dubois, H. Gouider, H. Prade. Possibilistic preference networks. Inf. Sci. 460-461, 401-415, 2018.

14. S. Benferhat, R. El Baida, F. Cuppens: A Possibilistic Logic Encoding of Access Control. Proc. 16th Int. Florida AI Research Soc. Conference (FLAIRS 2003), AAAI Press : 481-485, 2003.

15. S. Benferhat, D. Dubois, L. Garcia, H. Prade. On the transformation between possibilistic logic bases and possibilistic causal networks. Int. J. Approx. Reason. 29(2), 135-173, 2002.

16. S. Benferhat, D. Dubois, S. Kaci, H. Prade. Possibilistic merging and distance-based fusion of propositional information. Annals Math. and Artif. Intellig. 34, 217-252, 2002.

17. S. Benferhat, D. Dubois, H. Prade. Nonmonotonic reasoning, conditional objects and possibility theory. Artificial Intelligence 92(1-2), 259-276, 1997.

18. S. Benferhat, D. Dubois, H. Prade. Practical handling of exception-tainted rules and independence information in possibilistic logic. Appl. Intellig. 9(2), 101-127, 1998.

19. S. Benferhat, D. Dubois, H. Prade. An overview of inconsistency-tolerant inferences in prioritized knowledge bases. In: Fuzzy Sets, Logic and Reasoning about Knowledge, 395-417. Kluwer, 1999.

20. S. Benferhat, D. Dubois, H. Prade. A computational model for belief change and fusing ordered belief bases. In: Frontiers in Belief Revision, (Williams, M. A., Rott, H., eds.), Kluwer, 109-134, 2001.

21. S. Benferhat, D. Dubois, H. Prade, M. A. Williams. A framework for iterated belief revision using possibilistic counterparts to Jeffrey's rule. Fundam. Inform. 99, 147-168, 2010.

22. C. Cayrol, D. Dubois, F. Touazi. Symbolic possibilistic logic: Completeness and inference methods. J. Log. Comput. 28(1), 219-244, 2018.

23. C. Cayrol, D. Dubois, F. Touazi. Possibilistic reasoning from partially ordered belief bases with the sure thing principle. IfCoLog J. of Logics and their Applications, 5(1): 5-40, 2018.

24. D. Ciucci, D. Dubois. A modal theorem-preserving translation of a class of three- valued logics of incomplete information. J. Appl. Non-Class. Log. 23, 321-352, 2013. 
25. D. Ciucci, D. Dubois. From Possibility Theory to Paraconsistency. In Jean-Yves Béziau, Mihir Chakraborty, Soma Dutta (Eds.), New Directions in Paraconsistent Logic, Springer Proceedings in Mathematics \& Statistics, vol. 152, Springer, 229-248, 2015.

26. D. Ciucci, D. Dubois, J. Lawry. Borderline vs. unknown: comparing three-valued representations of imperfect information. Int. J. of Approximate Reasoning, 55, 1866-1889, 2014.

27. L. J. Cohen.The Probable and the Provable. Clarendon, Oxford, 1977.

28. D. Dubois. Belief structures, possibility theory and decomposable measures on finite sets. Computers and AI, 5, 403-416, 1986.

29. D. Dubois, L. Fariñas del Cerro, A. Herzig, H. Prade. A roadmap of qualitative independence. In: Fuzzy Sets, Logics and Reasoning about Knowledge, 325-350. Kluwer, 1999.

30. D. Dubois, E. Kerre, R. Mesiar, H. Prade, Fuzzy interval analysis. In: Fundamentals of Fuzzy Sets, (D. Dubois, H. Prade, eds.), Kluwer, Boston, Mass., 483-581, 2000.

31. D. Dubois, J. Lang, H. Prade. Possibilistic logic. In: Handbook of Logic in Artificial Intelligence and Logic Programming (Gabbay, D. M. et al. eds.), 3, 439-513, Oxford U. P., 1994.

32. D. Dubois, D. Le Berre, H. Prade, R. Sabbadin. Using possibilistic logic for modeling qualitative decision: ATMS-based algorithms. Fundamenta Informaticae, 37, 1-30, 1999.

33. D. Dubois, E. Lorini, H. Prade. The strength of desires: A logical approach. Minds \& Mach. 27(1), 199-231, 2017.

34. D. Dubois, H. Prade. Possibility Theory. An Approach to Computerized Processing of Uncertainty. With the collaboration of H. Farreny, R. Martin-Clouaire, C. Testemale. Plenum, New York, 1988.

35. D. Dubois, H. Prade. Epistemic entrenchment and possibilistic logic. Artificial Intelligence, 50, 223-239,1991.

36. D. Dubois, H. Prade. Possibility theory: qualitative and quantitative aspects. In: Handbook of Defeasible Reasoning and Uncertainty Management Systems (Gabbay, D., Smets, P., eds.), vol. 1, 169-226. Kluwer Academic, 1998.

37. D. Dubois, H. Prade. An overview of the asymmetric bipolar representation of positive and negative information in possibility theory. Fuzzy Sets \& Syst. 160(10), 1355-1366, 2009.

38. D. Dubois, H. Prade. Possibility theory and formal concept analysis: Characterizing independent sub-contexts. Fuzzy Sets \& Syst. 196, 4-16, 2012.

39. D. Dubois, H. Prade. Possibilistic logic. An overview. In. Handbook of The History of Logic. Vol. 9 Computational Logic (J. Siekmann, ed.) 283-342. North-Holland, 2014.

40. D. Dubois, H. Prade. Possibility theory and its applications: Where do we stand? Springer Handbook of Computational Intelligence (J. Kacprzyk, W. Pedrycz, eds.), Springer, 31-60, 2015.

41. Dubois, D., H. Prade. A crash course on generalized possibilistic logic. Proc. 12th Int. Conf. Scalable Uncert. Management (SUM'18), Milan. Springer LNCS, 11142, 3-17, 2018.

42. D. Dubois, H. Prade. Possibilistic logic: From certainty-qualified statements to two-tiered logics - A prospective survey. Proc. 16th Europ. Conf. on Logics in Artificial Intelligence (JELIA'19), (F. Calimeri, N. Leone, M. Manna, eds.), Rende, Italy, May 7-11, Springer LNCS 11468, 3-20, 2019.

43. D. Dubois, H. Prade. From possibilistic rule-based systems to machine learning - A discussion paper. Proc. 14th Int. Conf. on Scalable Uncertainty Management (SUM'20), (Davis, J., Tabia, K., eds.), Bozen-Bolzano, Sept. 23-25, Springer LNCS 12322, 35-51, 2020.

44. D. Dubois, H. Prade, R. Sabbadin. Decision-theoretic foundations of qualitative possibility theory. Europ. J. of Operational Research 128(3), 459-478, 2001.

45. D. Dubois, H. Prade, S. Schockaert. Stable models in generalized possibilistic logic. Proc. 13th Int. Conf. onPrinciples of Knowledge Representation and Reasoning (KR'12) (G. Brewka, T. Eiter, S. A. McIlraith, eds.), AAAI Press, 520-529, 2012. 
46. D. Dubois, H. Prade, S. Schockaert. Generalized possibilistic logic: Foundations and applications to qualitative reasoning about uncertainty. Artif. Intell. 252, 139-174, 2017.

47. P. Gärdenfors. Knowledge in Flux. MIT Press, 1988. 2nd ed. College Publications, 2008.

48. O. Gasquet, A. Herzig, B. Said, F. Schwarzentruber: Kripke's Worlds - An Introduction to Modal Logics via Tableaux. Studies in Universal Logic, Birkhäuser 2014.

49. J. Hintikka, Knowledge and Belief: An Introduction to the Logic of the Two Notions, Cornell University Press, 1962.

50. O. Kuzelka, J. Davis, and S. Schockaert. Encoding Markov logic networks in possibilistic logic. Proc. 31st Conf. on Uncertainty in Artificial Intelligence (UAI'15), (M. Meila and T. Heskes, eds.), Amsterdam, July 12-16, AUAI Press, 454-463, 2015.

51. O. Kuzelka, J. Davis, S. Schockaert. Induction of interpretable possibilistic logic theories from relational data. Proc. IJCAI'17, Melbourne, 1153-1159, 2017.

52. C. Lafage, J. Lang, R. Sabbadin. A logic of supporters. In: Information, Uncertainty and Fusion, (Bouchon-Meunier, B., Yager, R. R., Zadeh, L.A., eds.), 381-392. Kluwer, 1999.

53. D. Lehmann, M. Magidor. What does a conditional knowledge base entail? Artificial Intelligence 55(1), 1-60, 1992.

54. D. L. Lewis, Counterfactuals. Oxford: Basil Blackwell, 1973.

55. S. Link, H. Prade. Relational database schema design for uncertain data. Inf. Syst. 84, 88110, 2019.

56. P. Nicolas, L. Garcia, I. Stephan, C. Lefèvre. Possibilistic uncertainty handling for answer set programming. Ann. Math. Artif. Intell. 47(1-2), 139-181, 2006.

57. J. C. Nieves, M. Osorio, U. Cortés. Semantics for possibilistic disjunctive programs. Proc. 9th Int. Conf. LPNMR'07, Tempe, LNCS, 4483, 315-320, Springer, 2007.

58. O. Pivert, H. Prade. A certainty-based model for uncertain databases. IEEE Trans. Fuzzy Syst., 23(4), 1181-1196, 2015.

59. G. Qi, K. Wang. Conflict-based belief revision operators in possibilistic logic. Proc. 26th AAAI Conf. on Artif. Intellig., Toronto, 800-806, 2012.

60. G. Qi, Q. Ji, J. Z. Pan,J. Du. Extending description logics with uncertainty reasoning in possibilistic logic. Int. J. Intell. Syst. 26(4), 353-381, 2011.

61. M. Richardson, P. M. Domingos. Markov logic networks. Machine Learning, 62(1-2), 107136, 2006.

62. M. Serrurier, H. Prade. Introducing possibilistic logic in ILP for dealing with exceptions. Artif. Intellig. 171(16-17), 939-950, 2007.

63. G. L. S. Shackle. Decision, Order and Time in Human Affairs, 2nd edition. Cambridge University Press, UK, 1961.

64. G. Shafer. A Mathematical Theory of Evidence. Princeton University Press, 1976.

65. P. Walley. Measures of uncertainty in expert systems. Artificial Intelligence 83,1-58, 1996.

66. L. A. Zadeh. Fuzzy sets as a basis for a theory of possibility. Fuzzy Sets and Systems, 1, 3-28, 1978.

67. J. Zhu, G. Qi, B. Suntisrivaraporn. Tableaux algorithms for expressive possibilistic description logics. Proc.IEEE/ACM int. Conf. Web Intelligence (WI'13), Atlanta, 227-232, 2013. 